Formal vs. Intuitive Reasoning 1

Running Head: FORMAL VS. INTUITIVE REASONING

Cultural Preferences for Formal versus Intuitive Reasoning

Ara Norenzayan

University of Illinois

Edward E. Smith

University of Michigan
Richard E. Nisbett

University of Michigan

Beom Jun Kim

Yonsei University, Seoul, Korea

Address correspondence to: Ara Norenzayan, Department of Psychology, University of Illinois at Urbana-Champaign, 603 East Daniel Street, Champaign, IL 61820. Email: norenzay@uiuc.edu;

Fax (217) 244-5876. 


\begin{abstract}
We examined cultural preferences for formal versus intuitive reasoning among East Asian (Chinese and Korean), Asian American, and European American university students. We investigated categorization (Studies 1 and 2), conceptual structure (Study 3), and deductive reasoning (Studies 3 and 4). In each study a cognitive conflict was activated between formal and intuitive strategies of reasoning. European Americans, more than Chinese and Koreans, set aside intuition in favor of formal reasoning. Conversely, Chinese and Koreans relied on intuitive strategies more than European Americans. Asian Americans' reasoning was either identical to that of European Americans, or intermediate. Differences emerged against a background of identical reasoning tendencies across cultures in the absence of conflict between formal and intuitive strategies.
\end{abstract}




\section{Cultural Preferences for Formal versus Intuitive Reasoning}

Consider the following problem: is the Pope a bachelor?

Two approaches suggest themselves to solve this "bachelor" problem, each producing a different answer. One possibility is an intuitive solution: our understanding of "bachelor" reflects similarity relations among people who are known to be bachelors. According to this intuitive approach, the Pope would not be seen as a bachelor. Alternatively, we may represent the concept of "bachelor" as a person who satisfies the rule, "unmarried, adult, male." Under this formal definition, the Pope, contrary to intuition, indeed would be a bachelor.

This "bachelor" problem illustrates an important theoretical distinction in the psychology of reasoning. According to this distinction, human thinking is guided by two separate classes of cognitive strategies that implement different computational principles. One can be described as intuitive, experience-based, or holistic, whereas the other can be described as formal, rule-based, or analytic (Evans \& Over, 1996; James, 1890; Neisser, 1963; Smith, Langston, \& Nisbett, 1992; Tversky \& Kahneman, 1983; for a recent review of the empirical evidence for this distinction, see Sloman, 1996). The former cognitive strategies are associative or similarity-based in nature, and their computations reflect temporal contiguity and statistical regularities among features. The latter recruit symbolic representations, have logical structure and variables, and their computations reflect rule application. In this paper we use the terms intuitive and formal to refer to these two distinct reasoning systems.

In recent years, a growing number of research programs in psychology have examined these two cognitive systems under the rubric of "dual process" theories of thinking, including deductive reasoning (Evans \& Over, 1996), categorization (Rips, 1989; Smith, Patalano, \& Jonides, 1998), analogical reasoning (Gentner \& Medina, 1998), decision making (Tversky \& 
Kahneman, 1983), belief formation (Gilbert, 1991), and social cognition (Chaiken \& Trope, 1999). These two cognitive systems coexist in individuals, interact with each other in interesting ways (e.g., Gentner \& Medina, 1998; Sloman, 1996), and occasionally may be in conflict and produce contradictory inferences, as in the above "bachelor" problem. Although the intuitive system tends to dominate, the relative influence of one system versus the other on reasoning has been found to be influenced by the nature of the task, by instructions that emphasize rulefollowing or deduction (Evans \& Over, 1999; Sloman, 1996), by the particular reasoning domain (Atran, 1990; Keil, 1994), as well as by individual differences (Stanovich, 1999).

Little is known about the operation of these two systems of reasoning across diverse cultural groups. People in all cultures are likely to possess both of these reasoning systems, but cultural variation may exist in their relative accessibility and use to the extent that different values are placed on these reasoning systems. Many scholars have documented cultural differences in the intellectual outlooks of East Asian and Western cultures (Fung, 1952; Lloyd, 1996; Liu, 1974; Nakamura, 1960/1988). An analytic mode of thought has been held to be more prevalent in Western cultural groups. This mode is characterized by decoupling of the object from its context, assigning the object to categories based on necessary and sufficient features, and a preference for using rules, including the rules of formal logic, to explain and predict the object's behavior. In contrast, a holistic mode of thought has been held to be more prevalent in East Asian cultural groups. This mode is characterized by attention to the context or field as a whole, a concern with relationships among objects and between the field and the object, and a preference for intuitive approaches, as well as "dialectical" reasoning, which seeks the "middle-way" between conflicting propositions. 
Recent evidence indicates that some of these differences in analytic vs. holistic outlooks find their counterparts in a number of thought processes of contemporary Westerners and East Asians (for a review, see Nisbett, Peng, Choi, \& Norenzayan, 2001; Nisbett \& Norenzayan, in press). For example, East Asians see social behavior as the outcome of person-context interactions, in contrast to the characteristic American view of social behavior as the unfolding of dispositions. As a result, East Asians are less prone to the fundamental attribution error (Ross, 1977), or the tendency to overattribute behavior to dispositions despite obvious situational constraints (Choi, Nisbett, \& Norenzayan, 1999). East Asians are more field dependent (Witkin \& Berry, 1975) than Americans, being more influenced by the position of the surrounding frame when judging the position of the rod in the rod-and-frame task (Ji, Peng, \& Nisbett, in press). East Asians have a more holistic sense of causality: they draw on a wider range of factors to explain events. As a result, East Asians experience less surprise at unexpected events and show more hindsight bias (Fischhoff, 1975), the tendency to view events as having been inevitable in retrospect (Choi \& Nisbett, 2000). East Asians also prefer dialectical resolutions to apparent contradictions, so that the contradiction is tolerated or a compromise solution is sought. Americans respond to contradiction by "polarizing" their opinions - deciding that one proposition is true and the other false (Peng \& Nisbett, 1999; Davis, Nisbett, \& Schwarz, 2000). In this paper, we examine whether analogous cultural differences can be found in formal and intuitive systems of reasoning.

\section{Selection of Participants}

Participants were self-identified European American, Asian American, and East Asian undergraduate students, who were otherwise similar in their demographic background and intellectual ability. In Studies 1 and 2, the East Asians were international students at the 
University of Michigan who were of Chinese or Korean culture, had lived in the US for less than 4 years, had graduated from a high school in East Asia, and grew up speaking an East Asian language. In Studies 3 and 4, East Asians were Korean students at Yonsei University in Seoul, Korea. All European Americans were students at the University of Michigan. Asian Americans grew up in the United States, and were of Chinese, Korean, or Japanese ethnic background. The Asian American samples were included to examine acculturation effects. Their thinking was expected to be intermediate between the two other groups, because of their substantial, though probably not complete, socialization into American traditional culture.

Theoretical Rationale for the Cultural Differences

In each study a simple research strategy was used to measure reasoning preferences. A cognitive conflict was activated such that formal thinking was pitted against intuitive thinking. If European Americans favor formal rules more than intuition, they should be more willing to set aside intuition and follow rules when the two are in conflict. If East Asians favor intuition more than formal rules, they should be less willing to abandon intuition in favor of formal rules.

We had a different prediction for reasoning based on intuition alone or on rules alone, with no cognitive conflict present. In these cases responses are a function of cognitive ability, rather than a function of preference for a particular cognitive strategy. Because we selected our participants in different cultures so that they were similar in cognitive abilities, we expected no cultural differences in their ability to implement a formal strategy or an intuitive strategy in the absence of a conflict between the two.

We examined the extent to which cultural differences might be found for a variety of cognitive tasks. In Study 1, we examined category learning. Participants were asked to apply a complex rule as a way of correctly classifying imaginary animals. Their category learning was then 
tested in a situation in which the complex rule conflicted with memory for previously seen animals. In Study 2, we investigated classification and similarity judgments of drawings (e.g., geometric objects, houses, flowers) that admitted either a rule-based judgment or a judgment based on family resemblance structure. Studies 3 and 4 went beyond immediately perceptible stimuli to examine conceptual processes. In Study 3, participants evaluated the convincingness of deductive arguments when logic conflicted with the typicality of the conclusion. Finally, in Study 4 we investigated deductive reasoning when logical structure was pitted against the believability of the conclusion.

\section{Study 1: Rule- vs. Exemplar-Based Category Learning}

If there is a cultural difference between European Americans and East Asians in formal and intuitive thinking, this difference might be apparent when categories have been learned by the formal application of rules, and when subsequent classification by rules conflicts with intuitive knowledge, such as exemplar memory. Rule-based categorization is accomplished by determining whether or not the novel object satisfies a rule that defines the category by its necessary and sufficient features, such as when a new neighbor is categorized as a bachelor if he satisfies the rule, "is an unmarried adult male." Exemplar-based categorization, in contrast, reflects similarity of the novel object to previously stored exemplars retrieved from memory. The more similar the new object and the retrieved exemplars are, the more likely it is that the new object belongs to the same category as the retrieved exemplars. Our new neighbor might be suspected of being a bachelor not because he satisfies some well-articulated rule, but because he is reminiscent of a bachelor relative.

Study 1 was based on a well-developed paradigm in categorization research (e.g. Allen \& Brooks, 1991; Regehr \& Brooks, 1993; Smith et al., 1998). Participants viewed imaginary 
animals on a computer screen and were told that the animals belonged to different categories, some being from Venus and others being from Saturn. In a training phase, participants learned to categorize, with feedback, the set of animals. This was followed by a test phase, in which participants were asked to categorize new animals. Participants in an exemplar memory condition, which served as a control condition, were asked during the training phase simply to observe a series of the cartoons and initially make guesses as to which animal belonged to which category. Feedback was given after each guess. As participants repeatedly categorized the same animals, they could rely on their memory of previous exemplars to assign the animals to their appropriate categories. Participants in the rule condition learned a complex rule, making categorizations based on whether or not the animal had three out of five specific bodily features.

Critically, in the test phase of the rule condition, half of the animals were positive matches: they belonged to one category by the rule, and also were very similar to a training exemplar from that same category. The other half of the new animals were negative matches: they belonged to one category as defined by the rule, but were very similar to a training exemplar from the opposite category (see Figure 1). Thus the negative match animals -- unlike positive match ones -- posed a cognitive conflict between rule-based and exemplar-based categorization.

Based on past findings (Allen \& Brooks, 1991; Smith, et al., 1998; Patalano, Smith, \& Jonides, 2001), we anticipated that: (1) participants would make fewer classification errors in the rule condition than in the exemplar memory condition, but take longer because of the necessity to compute the explicit rule, and (2) participants in the rule condition would make more classification errors and show slower reaction times for negative matches than positive matches, that is, when rule and exemplar memory were in conflict than when they were not. 
Most importantly, the cultural analysis led to the prediction that (3) the difference in classification errors between positive- and negative-matches would be greater for East Asians than for European Americans, since East Asians would be more inclined to use memory for the exemplars in the rule condition. Similarly, the difference in response times between positive- and negative-matches was expected to be greater for East Asians.

\section{Method}

\section{$\underline{\text { Participants }}$}

Participants were 61 European American (27 men, 34 women), 61 Asian American (32 men, 29 women), and 28 East Asian (12 men, 16 women) undergraduate students at the University of Michigan (age $\underline{\mathrm{M}}=19$ ) who received partial course credit for their participation.

\section{$\underline{\text { Stimuli }}$}

The stimuli were color drawings of imaginary animals (from Patalano et al, 2001; see

Figure 1 for examples). The animals were constructed out of 10 binary features: ears (antennae ears or regular ears), tail (curly or straight), neck length (long or short), mouth (open mouth or snout), feet (hooves or webbed feet), leg length (short or long), head direction (pointed up or down), body color (red or blue), body marking (spots or stripes), and body shape (round or angular). The animals were categorized as being from Venus or Saturn by a 5-feature additive rule that used ears, tail, neck length, mouth, and feet as diagnostic features. An animal was defined as being from Venus if it satisfied at least 3 of the 5 diagnostic features; otherwise it was from Saturn. All Venus animals always satisfied exactly 3 of the 5 diagnostic features; all Saturn animals satisfied only 2 of the 5 diagnostic features.

Ten different animals were presented 4 different times in the training phase, followed by 20 new animals in the test phase, consisting of a positive match and a negative match to each 
animal in the training phase. The design was counterbalanced, such that half of the animals were from Venus, and the other half were from Saturn. As can be seen in Figure 1, a positive match was very similar to its corresponding training exemplar, varying only on one of the irrelevant features (e.g., head pointed up or down). In contrast, a negative match was also very similar to its corresponding training exemplar, but varying this time only on one of the features diagnostic of the rule (e.g., antennae or regular ears). This made it possible for animals to change categories, yet remain similar to an item in the opposite category. The values of the irrelevant features appeared equally often in each category, and thus were not diagnostic of category membership. $\underline{\text { Procedure }}$

Participants were tested individually on a computer. All the instructions and materials were in English, as all participants, including the East Asian students, were enrolled at an American university and were fluent in English.

Participants from the three cultural groups were randomly assigned to either the rule condition or the exemplar memory condition, which served as an experimental control. Participants in the rule condition were given the rule at the beginning of training, and they memorized it before beginning the study (post-study interviews indicated that all participants in the rule group had successfully memorized the rule). Those in the exemplar-memory condition were never given a rule. They were instructed to guess whether the animal was from Venus or Saturn the first time they saw an animal, and subsequently categorize new ones by using their knowledge of previously studied animals. Prior to being presented with the actual animals, participants practiced with one sample animal as a way to familiarize themselves with the task.

In the training phase participants were given a total of 40 trials, consisting of 10 different animals categorized 4 times each, 1 animal at a time, each participant receiving them in 1 of 4 
different random orders. Participants were instructed to categorize the animals as quickly as they could, without sacrificing accuracy. They received feedback after each categorization decision, which they made by pressing a designated button on the keyboard. They then pressed another designated button to go to the next stimulus screen. The training phase was identical in the rule and exemplar memory conditions, except that rule participants used the rule.

The test phase was also identical for both experimental groups, with the same exception that rule participants continued to use the rule. Each training animal corresponded to one positive match and one negative match animal in the test phase. Unlike in the training phase, no feedback was given in the test phase. Otherwise the procedures in both phases were identical.

The dependent measures were: the percentage of classification errors, and the reaction times for accurate responses in the training and test phases.

\section{$\underline{\text { Results }}$}

\section{$\underline{\text { Training Phase }}$}

The data in the training phase established that all participants in both the rule and exemplar memory conditions learned to categorize the animals, the learning curves being similar for the three cultural groups. In the rule condition, a culture by trials analysis of variance (ANOVA) revealed a linear decrease of classification errors across the four trials, $\underline{F}(3,201)=6.43, \underline{p}<.001$, with no main effect of culture, $\underline{\mathrm{F}}(2,67)=1.79$, $\underline{\mathrm{ns}}$, or learning by culture interaction, $\underline{\mathrm{F}}(6,201)=$ 1.35 , ns. Misclassifications, averaged across the three cultural groups, decreased from $20.7 \%$ (first trial) to $11.3 \%$ (last trial). Similarly, reaction times decreased linearly across the four trials, $\underline{\mathrm{F}}(3,201)=42.30, \underline{\mathrm{p}}<.001(\underline{\mathrm{M}}=5586 \mathrm{~ms}$, first trial vs. $\underline{\mathrm{M}}=3294 \mathrm{~ms}$ last trial $)$. There was no main effect of culture, $\underline{\mathrm{F}}<1$, or learning by culture interaction, $\underline{\mathrm{F}}<1$. 
Results were similar in the exemplar-memory condition. Classification errors decreased linearly across the four trials, $\underline{\mathrm{F}}(3,231)=10.49, \underline{\mathrm{p}}<.001$, down from $51.9 \%$ (first trial), to $39.9 \%$ (last trial), which was significantly better than chance $(50 \%), \underline{t}(79)=4.62, \underline{p}<.001$. There was no main effect of culture, $\underline{\mathrm{F}}<1$, or culture by learning interaction, $\underline{\mathrm{F}}<1$. Reaction times also revealed a main effect of learning across the 4 trials, $\underline{\mathrm{F}}(3,231)=7.88, \underline{\mathrm{p}}<.001$, down from $\underline{\mathrm{M}}=$ $1883 \mathrm{~ms}$ (first trial), to $\underline{\mathrm{M}}=1431 \mathrm{~ms}$ (last trial), with no main effect of culture, $\underline{\mathrm{F}}<1$, or learning by culture interaction, $\underline{\mathrm{F}}<1$.

Test Phase: Rule Group versus Exemplar-Memory Group

Thus the three cultural groups in each condition learned to categorize successfully and entered the test phase on an equal footing. Based on prior research, we expected that participants in the rule condition would make fewer classification errors and have slower reaction times than participants who instead had to rely on exemplar memory. An instruction (rule vs. exemplar memory) by culture (European American, Asian American, East Asian) by match (positive vs. negative, repeated measure) ANOVA indicated that this was indeed the case, with a main effect for classification errors, $\underline{F}(1,144)=507, \underline{p}<.001$, and for reaction times, $\underline{F}(1,144)=124.30, \underline{p}<$ .001 .

\section{Test Phase: Exemplar-Memory Group}

Categorization decisions were analyzed separately for participants who were given the rule and for those who had to rely on exemplar memory, the latter serving as an experimental control for the cultural predictions. The results for the exemplar-memory condition for classification errors and reaction times are shown in Figure 2. For the negative match items the classification error rate was reverse-scored, since in the absence of the rule the only correct response would be to categorize based on similarity to past exemplars. As can be seen, participants' categorization 
was significantly better than chance (i.e., 50\%) for positive matches, $\underline{\mathrm{t}}(79)=6.58, \underline{\mathrm{p}}<.001$, and for negative matches $\underline{t}(79)=8.16, \underline{p}<.001$. No cultural differences emerged for either classification errors or reaction times, $\underline{\mathrm{F}}(2,77)=1.18, \underline{\mathrm{ns}}$, and $\underline{\mathrm{F}}<1$, respectively. All cultural groups learned the categories using exemplar memory equally well.

\section{Test-Phase: Rule Group}

Consistent with prior research, a repeated-measures ANOVA for the rule group indicated that participants overall made more classification errors when the rule was in conflict with exemplar memory (negative matches), than when there was no such conflict (positive matches), $\underline{F}(1,67)=20.04, \underline{p}<.001$. A similar main effect emerged for reaction times, with slower reaction times for negative than positive matches, $\underline{\mathrm{F}}(1,67)=16.57, \underline{\mathrm{p}}<001$ (see Figure 3 ).

Most interestingly, the predicted cultural differences in the degree of classification errors for negative as opposed to positive matches was obtained: there was a significant culture by item type interaction, $\underline{F}(2,67)=3.81, \underline{p}<.05$. Analyses of partial interactions indicated that, as expected, the negative-positive difference was larger for East Asians than European Americans, $\underline{F}(1,67)=4.97, \underline{p}<.05$, and it was also larger for East Asians than Asian Americans, $\underline{F}(1,43)=$ $7.49, \underline{p}<.01$ (see top panel of Figure 3 ). As to reaction times, there was a main effect of culture, $\underline{F}(2,67)=3.43, \underline{p}<.05$, but no culture by item type interaction, $\underline{\mathrm{F}}<1$. Analyses of simple effects revealed that East Asians had slower reaction times than European Americans, $\underline{F}(1,67)=6.15, \underline{p}$ $<.02$, and Asian Americans, $\underline{\mathrm{F}}(1,67)=3.86, \underline{\mathrm{p}}=.05$ (see bottom panel of Figure 3).

Finally, two analyses were conducted to determine whether differences in English language fluency could account for the cultural differences in category learning. Because most East Asians were not native English speakers, and the study was conducted in English, it is conceivable that their lesser fluency could have contributed to a weaker tendency to apply the 
rule, especially when the rule was in conflict with exemplar memory. This idea was tested in two ways: (1) the rule group's reported verbal SAT scores were correlated with their performance on the negative match items, and (2) East Asian and Asian American participants in the rule condition were pooled into a single sample (European Americans were excluded, because they were all native English speakers), and separated by their reported native language (whether or not they grew up speaking English or an East Asian language). If language fluency was a factor in their performance, those who reported an East Asian language to be their native language should have had higher error rates and slower reaction times for negative matches.

There was no evidence that the category learning results were influenced by English language fluency. The correlations between verbal SATs and performance on negative match items for the rule group were no different from zero, either for classification errors, $\underline{\mathrm{r}}(58)=-.07$, or for reaction times, $\underline{\mathrm{r}}(58)=.04$ (11 participants failed to report their SAT scores). Nor did reported native language matter. Participants who grew up speaking an East Asian language made no more classification errors than native English speakers $(\underline{\mathrm{M}}=16.25$ vs. $\underline{\mathrm{M}}=17.14), \underline{\mathrm{t}}<1$, nor did they have slower reaction times $(\underline{\mathrm{M}}=3806$ vs. $\underline{\mathrm{M}}=4207), \underline{\mathrm{t}}(35)=1.08$, $\underline{\mathrm{ns}}$.

\section{$\underline{\text { Discussion }}$}

Our central prediction was that a cultural difference would emerge in the extent to which memory for exemplars would interfere with rule application when the two processes were in conflict. The results supported this prediction. East Asians and European Americans categorized equally well in the exemplar memory condition. In the rule condition, however, European Americans and Asian Americans made fewer classification errors than East Asians for negative matches - for which a conflict existed between rule- and exemplar-based categorization. No differences emerged for the positive matches -- for which no such conflict existed. 
Similarly, no cultural differences in response times emerged in the exemplar memory condition, although East Asians' response times in the rule condition were overall slower than those of European Americans. One possible explanation for this finding is that there might be an important difference between classification errors and reaction times. Since positive matches were randomly intermixed with negative matches, participants who were particularly sensitive to exemplar memory might have expected a conflict at any trial. Thus rule application might have been harder for East Asians, in a context where the rule could conflict with exemplar-based categorization for any given trial. Finally, Asian Americans responded identically to European Americans in all respects.

\section{Study 2: Rule- vs. Family Resemblance-Based Classification and Similarity Judgments}

Study 1 demonstrated that category learning based on the application of a formal rule was easier for Americans than for East Asians when the rule conflicted with exemplar memory. However, exemplar-based reasoning is only one strategy among many intuitive strategies, another being family resemblance. This strategy draws on knowledge of graded membership of exemplars within a category, such that some are believed to be more similar to the category than others (Rosch, 1978; Rosch \& Mervis, 1975; Wittgenstein, 1953).

If Western thinking is more rule-governed and less intuitive than East Asian thinking, Westerners may be more tempted to spontaneously locate objects in novel categories based on a simple rule, and less willing to rely on family resemblance, whereas the reverse might be true for East Asians. Study 2 tested this hypothesis.

Participants placed objects in one of two categories that could be defined on the basis of a unidimensional rule or overall similarity (Kemler-Nelson, 1984). They saw a series of presentations, each consisting of a "target" object beneath two groups or categories of four 
similar objects, as illustrated in Figure 4. In one condition, participants were asked to decide which category the target object belonged to (the classification condition). Such free classification has been shown to be unidimensional, as long as the stimuli allow scanning of an array of dimensionalized objects, as was the case in this study (Ahn \& Medin, 1992; Medin, Wattenmaker, \& Hampson, 1987). To encourage more strongly the use of an alternate family resemblance strategy, other participants were instructed to judge which category the target object was most similar to (the similarity judgment condition). The stimuli were constructed such that the responses driven by the rule vs. family resemblance criterion led to different decisions. Participants could rely on a unidimensional rule, deciding whether or not the target object shared a single feature with all category members. Alternatively, they could rely on family resemblance, judging the target object to be holistically similar to all members of a category because it shared a large number of features with them, even though no one feature characterized all members of the category. The anticipation was that East Asians would make less use of rules and more use of family resemblance than would European Americans in both conditions. As to response times, it was expected that the similarity instructions would produce faster response times than the classification instructions. However, because making judgments based on a unidimensional rule or on family resemblance are equally fast, there was no reason to expect cultural differences in reaction times in this study.

\section{Method}

\section{Participants}

Participants were undergraduate students at the University of Michigan (age $\underline{\mathrm{M}}=19$ ). The sample consisted of 52 European Americans (27 men, 25 women), 52 Asian Americans (28 men, 24 women), and 53 East Asians of Chinese and Korean ethnic background (27 men, 26 women). 
$\underline{\text { Stimuli }}$

Figure 4 shows examples of the stimulus sets used. Each one had an abstract structure, described in Table 1, constructed from a set of 4 binary features. Each stimulus set corresponded to one item (e.g., flower, house), and was composed of a target object and two categories (Group 1 and Group 2), instantiated as four objects belonging to each category. The two categories and the target object for each stimulus set were designed so that two alternative solutions were possible. One of the four binary features always defined each category (e.g., all four instantiations of the flower in one group had a short stem and all four in the other had a long stem). The other three, non-deterministic features took on a different value for each category, such that each of the four objects in each category contained 3 out of the 4 values, which together produced a strong family resemblance structure that separated the two categories based on overall similarity. A rulebased solution would select the deterministic feature that the target object shared with one of the categories; a family-resemblance solution would select overall similarity of the target object to the category members. Ten category pairs were used, each pair shown with 1 of the 2 alternative targets, counterbalancing the design, and yielding a total of 20 stimulus sets. $\underline{\text { Procedure }}$

Participants were tested individually on a computer. All the instructions and materials were in English, as all participants, including the East Asian students, were enrolled at an American university and were fluent in English. Participants were randomly assigned to the classification or the similarity judgment condition. In the classification condition, participants were asked, for each stimulus set, to decide "which group the target object belongs to." In the similarity judgment condition, other participants were instructed to decide "which group the target object is most similar to." Before seeing the actual stimuli, participants practiced with one sample stimulus 
set as a way to be familiarized to the task. At this point the experimenter verified that the participant understood the instructions and was ready to begin. The stimulus sets were then presented to participants in a random order. The computer program automatically moved to the next screen as soon as a response was made. Participants were asked "to take their time while responding, but not spend too much time on any single item.”

For each stimulus set, participants indicated their classification or similarity judgment decision by pressing a designated button. The dependent measure was the percentage of rule and family resemblance solutions for each participant, averaged across the 20 trials. Reaction times (RT) were also recorded. Participants who were instructed to make categorization decisions were expected to be slower than those who were asked to judge overall similarity (Smith, et al., 1998).

\section{$\underline{\text { Results }}$}

The results are shown separately for the classification condition and the similarity judgment condition. As seen in the top panel of Figure 5, participants overwhelmingly preferred to classify based on the unidimensional rule rather than family resemblance, replicating past research (e.g., Medin, et al., 1987), $\underline{\mathrm{F}}(1,100)=44.40, \underline{\mathrm{p}}<.001(\underline{\mathrm{M}}=67 \%$ vs. $\underline{\mathrm{M}}=33 \%)$. Contrary to predictions, there was no culture by response type interaction, $\underline{\mathrm{F}}(2,100)=1.25$, $\underline{\mathrm{ns}}$. All three cultures substantially preferred rule-based over family resemblance classification, $\underline{\mathrm{t}}(33)=$ 4.23, $\underline{\mathrm{p}}<.001$ for European Americans, $\underline{\mathrm{t}}(33)=2.48, \underline{\mathrm{p}}<.02$ for Asian Americans, and $\underline{\mathrm{t}}(34)=$ 4.94, $\mathrm{p}<.001$ for East Asians.

Matters were different when participants were asked to judge the similarity of the target object to the categories, as may be seen in the bottom panel of Figure 5. Under these instructions, a marked cultural difference emerged. There was a significant culture by response type interaction, $\underline{F}(2,51)=8.01, \underline{p}<.005$, and no main effect of response type, $\underline{\mathrm{F}}<1$. European 
Americans gave many more responses based on the unidimensional rule than on family resemblance, $\underline{\mathrm{M}}=69 \%$ vs. $\underline{\mathrm{M}}=31 \%$ ), $\underline{\mathrm{t}}(17)=3.68, \underline{\mathrm{p}}<.005$ (Indeed, they preferred the unidimensional rule precisely to the same extent as when making classification decisions.) East Asians, in contrast, gave fewer rule-based responses than family resemblance responses, $\mathrm{t}(17)=$ $2.09, \underline{p}=.05,(\underline{M}=41 \%$ vs. $\underline{M}=59 \%)$. Asian Americans were intermediate, having no preference for rule vs. family resemblance responses $(\underline{\mathrm{M}}=46 \%$ vs. $\underline{\mathrm{M}}=54 \%), \underline{\mathrm{t}}<1$.

Reaction times were submitted to a culture by instruction condition (classification vs. similarity judgment) ANOVA. Only a main effect of judgment condition emerged, indicating that participants overall were slower when classifying than when judging similarity (in milliseconds, $\underline{\mathrm{M}}$ $=7516, \underline{\mathrm{SD}}=3160$, vs. $\underline{\mathrm{M}}=6260, \underline{\mathrm{SD}}=4379), \underline{\mathrm{F}}(1,151)=4.25, \underline{\mathrm{p}}<.05$. There were no main effects of culture, $\underline{\mathrm{F}}<1$, or culture by judgment condition interaction, $\underline{\mathrm{F}}<1$.

\section{Study 3: Conceptual Structure Based On Logic vs. Typicality}

The first two studies probed categorization and similarity judgments of artificially constructed categories. In contrast, in Study 3 we examined the extent to which people spontaneously rely on formal rules versus intuition to mentally represent naturally occurring categories. This was done by setting logic against the typicality of category exemplars. Typicalitybased reasoning relies on the similarity relations among particular exemplars of a category, with typicality judgments usually (but not always) being a function of the number of features shared by other category members (Rosch \& Mervis, 1975; Smith \& Medin, 1981). For example, penguins are atypical birds because of their perceptual peculiarities - large body, small wings, no flying ability -- that set them apart from other members of the category "bird." This type of reasoning is intuitive in that it relies on the perceptual features of actual category members, or on second-hand knowledge of the perceptual features of exemplars of a category. 
In Study 3, we assessed reasoning preferences based on logic versus typicality, investigating how people project fictitious or unknown features from a superordinate category (e.g., bird) to subordinate categories of varying typicality (e.g., eagle, penguin) (Sloman, 1993). Participants rated how convincing they found deductive categorical arguments such as:

1. All birds have ulnar arteries

Therefore all eagles have ulnar arteries

2. All birds have ulnar arteries

Therefore all penguins have ulnar arteries

There are two known strategies one can recruit to reason about these arguments -- one formal, and the other intuitive. Reasoners following logic would "discover" the hidden premise in each argument, that "All eagles are birds," and "All penguins are birds.” Once these hidden premises are exploited, the argument becomes a standard valid deductive argument. Armed with this knowledge, participants should be equally convinced by the typical and atypical arguments.

But the typicality of the conclusion category can affect people's intuitions about the convincingness of arguments to the extent that they are inclined to ground their reasoning in intuition rather than logic. Sloman (1993) found that when both typical and atypical arguments were evaluated by every participant (thus making typicality salient), American students showed a typicality effect, being less convinced of atypical arguments than typical ones.

Study 3 examined this typicality effect cross-culturally. The phenomenon was evaluated both within-groups (when typicality information is salient), and between-groups (when typicality information is not salient). If East Asians are more likely than European Americans to rely on intuition when it conflicts with logic, the typicality effect should be stronger for East Asians than for European Americans, particularly when typicality is not made salient. 


\section{$\underline{\text { Participants }}$}

\section{$\underline{\text { Method }}$}

Ninety-two European American students (40 men, 52 women, age $\underline{M}=19)$ and 74 Asian American students at the University of Michigan (35 men, 39 women, age $\underline{M}=19$ ), as well as 93 Korean students at Yonsei University in Seoul, Korea (49 men, 44 women, age $\underline{M}=23$ ) participated for partial course credit. In addition, a separate group of 42 European American students at Michigan (16 men, 26 women, age $\underline{M}=19)$ and 42 Korean students at Yonsei (26 men, 16 women, age $\underline{M}=22$ ) rated the typicality of the conclusions as a manipulation check.

\section{$\underline{\text { Materials }}$}

Materials were generated in collaboration with two bilingual Korean researchers to ensure the functional equivalence of the materials. Care was taken to generate categories that were equally familiar to Americans and Koreans, and having conclusion categories that were equally typical or atypical in these cultures. Ten pairs of arguments were constructed using this procedure. For each argument pair, the premise superordinate category was held constant, and the typicality of the conclusion subordinate category was varied, with an unknown or a fictitious feature being projected from the former to the latter category. Half of the arguments had typical conclusions and the other half had atypical ones (see Table 2 for examples).

\section{$\underline{\text { Procedure }}$}

The study was conducted in a classroom setting. Participants received a questionnaire packet containing all the instructions and materials. They were instructed to evaluate the convincingness of each argument on an 11-point scale:

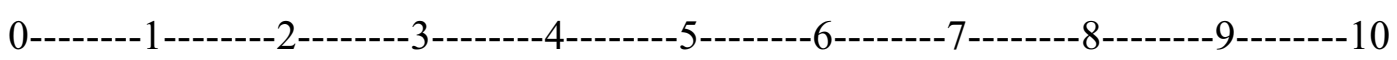
Very Unconvincing/Very Weak
Very Convincing/Very Strong 
Because Study 3 was concerned with the spontaneous reliance on logic versus conclusion typicality, the instructions did not provide any clues to respond logically to the arguments; instead, participants were asked to "read each argument, assume that the facts given to you are true, and using the scale, evaluate how convincing you believe each argument is."

After responding to all 20 arguments, participants completed a brief demographic questionnaire. They were given as much time as they needed, with all participants completing the questionnaire within 10 minutes. For Korean participants, all materials were translated into Korean using the back-translation method (Brislin, 1970).

Salience of Typicality Manipulation

In contrast to previous studies of this phenomenon which have relied on an exclusively within-groups methodology which makes typicality a salient feature of the task, the present study manipulated the salience of typicality by counterbalancing the order of presentation of the typical and atypical arguments. Half of the participants in each cultural group were randomly assigned to evaluate all 10 typical arguments first, followed by all 10 atypical arguments (typical first condition). The other half was randomly assigned to evaluate all 10 atypical arguments first, followed by all 10 typical arguments (atypical first condition). Thus, the typicality effect was evaluated in two ways: as a between-groups comparison of the first 10 typical versus atypical arguments (low salience of typicality). The typicality effect was also measured as a betweengroups comparison of the last 10 typical and atypical arguments (high salience of typicality).

\section{Manipulation Check}

A separate group of European American and Korean students rated the typicality of the conclusion categories to establish the effectiveness of the typicality manipulation and the functional equivalence of the typicality information across cultures. Participants rated the 
typicality of the conclusions for each of the 20 arguments (for example, "how typical are eagles of birds?") on a scale anchored between 0 (Not at all typical) and 10 (Very typical). The items were counterbalanced so that half rated typical ones first, and half rated atypical ones first.

\section{$\underline{\text { Manipulation Check }}$}

\section{$\underline{\text { Results }}$}

The typicality manipulation was successful. All typical conclusions were rated as more typical than their atypical counterparts, for both European Americans and Koreans. Averaging across the ten typical and ten atypical conclusions, the effect was highly reliable, for European Americans, $\underline{\mathrm{t}}(42)=12.71, \underline{\mathrm{p}}<.001$, typical $\underline{\mathrm{M}}=8.32, \underline{\mathrm{SD}}=1.69$, atypical $\underline{\mathrm{M}}=5.24, \underline{\mathrm{SD}}=2.15$, and for Koreans, $\underline{\mathrm{t}}(42)=17.18, \underline{\mathrm{p}}<.001$, typical $\underline{\mathrm{M}}=8.49, \underline{\mathrm{SD}}=1.18$, atypical $\underline{\mathrm{M}}=5.00, \underline{\mathrm{SD}}=$ 1.45. There was no culture by conclusion typicality interaction, $\underline{F}(1,82)=1.61$, ns.

\section{Convincingness of Arguments}

We examined the results in a between-groups design separately where there was (1) low salience of typicality (the first 10 arguments), and (2) high salience of typicality (the last 10 arguments).

Low Typicality Condition. The top panel of Figure 6 shows the convincingness ratings when typicality salience was low (the first 10 arguments), allowing participants elbow room to set aside typicality and follow logic. A culture (European American, Asian American, Korean) by argument type (typical, atypical) between-groups ANOVA revealed a main effect of argument type, as an indicator of the typicality effect, $\underline{F}(1,253)=25.76, \underline{p}<.001$. As predicted, a culture by argument type interaction emerged, reflecting differing strengths of the typicality effect for Koreans, European Americans, and Asian Americans, $\underline{F}(2,253)=4.67, \underline{p}<.01$. Analysis of partial interactions indicated that, as predicted, the typicality effect was stronger for Koreans than 
European Americans, $\underline{\mathrm{F}}(1,253)=9.09, \underline{\mathrm{p}}<.005$, and it was marginally stronger for Koreans than Asian Americans, $\underline{\mathrm{F}}(1,253)=3.46, \underline{\mathrm{p}}=.06$. European Americans and Asian Americans did not differ, $\underline{F}<1$.

The cultural differences were most revealing when the typicality effect was evaluated separately for each cultural group. As expected, a large typicality effect emerged for Koreans, $\underline{\mathrm{t}}(91)=5.98, \underline{\mathrm{p}}<.001$. It was marginally significant for Asian Americans, $\underline{\mathrm{t}}(72)=1.92, \underline{\mathrm{p}}=.06$. Most tellingly, European Americans did not show the typicality effect, $\underline{t}(90)=1.16, \underline{p}=n s$. Instead, their responses were consistent with logic, being equally convinced by both arguments. High Typicality Salience condition. The bottom panel of Figure 6 presents the results when salience of typicality was high (last 10 arguments). Reflecting the typicality effect, a culture (European American, Asian American, Korean) by argument type (typical, atypical) betweengroups ANOVA revealed a main effect of argument type, with typical arguments being more convincing than atypical ones, $\underline{F}(1,253)=94.59, \underline{p}<.001$. There was no culture by argument type interaction, $\underline{F}<1$. Thus, all 3 groups showed the typicality effect to the same extent when the salience of typicality was high.

Finally, the results were reexamined with typicality as a within-groups manipulation. In this design, the expected cultural difference in the typicality effect emerged again, culture (European American, Asian American, Korean) by argument type (typical, atypical) interaction, $\underline{F}(2,256)=5.49, \underline{p}<.005$. An analysis of partial interactions revealed that the typicality effect was larger for Koreans than for European Americans, $\underline{F}(1,256)=10.82, \underline{p}<.005$; it was (marginally) larger for Asian Americans than European Americans, $\underline{F}(1,256)=3.62, \underline{p}=.06$; but did not differ in magnitude between Koreans and Asian Americans, $\underline{F}(1,256)=1.44, \underline{p}>.20$. 


\section{Study 4: Logic vs. Belief in Deductive Reasoning}

Evidence presented in Studies 1-3 indicates that the divergent intellectual traditions of East Asia and the West may be reflected in the ways contemporary East Asians and Westerners categorize and organize concepts. In Study 4 we examined the consequences of this cultural difference for reasoning based on logical structure vs. empirical belief. Unlike Study 3, which did not directly manipulate the logical structure of arguments, Study 4 included an orthogonal variation of both the logical structure of arguments and the intuitive content of the conclusions.

Although cognitive psychologists have rarely examined the cultural bases of logical reasoning, there exists one such program, rooted in the Vygotskian tradition, and developed by early cultural psychologists (Cole, Gay, Glick, \& Sharp, 1971; Luria, 1971; Scribner, 1977). The central finding of this research, conducted among non-Western cultures as diverse as Uzbek farmers in the former Soviet Union, the Kpelle of West Africa, and the Maya in Mexico, has been the participants' unwillingness to decouple the content of the deductive problem from its logical structure. For example, a Kpelle man was given the following problem: All Kpelle men are rice farmers/Mr. Smith is not a rice farmer/Is he a Kpelle man? The man's response, characteristic of many of these participants, was, "I don't know the man in person. I have not laid eyes on the man himself." (Scribner, 1977, p. 490). Thus, non-Westerners in these studies typically refused to solve deductive problems, on the grounds that the content is unfamiliar or contrary to experience. This suggests that knowledge may counter logic for non-Westerners to a greater extent than for Westerners, especially when the two are in conflict.

In Study 4, such a cognitive conflict was created between the logical structure of a deductive argument and the empirical plausibility of the argument's conclusion. Participants evaluated the logical validity of a series of categorical syllogisms and conditional arguments that 
were either valid or invalid and that had conclusions that were either believable or nonbelievable. Moreover, at the end of the task, the same argument forms were presented in an abstract version so as to assess logical reasoning independent of content. Finally a separate group of participants rated the believability of each conclusion as a manipulation check and in order to establish the functional equivalence of the believability manipulation across cultures.

To the extent that one's reasoning is guided by intuitive knowledge, the believability of the conclusion may interfere with logical evaluations. As a result, valid arguments with implausible conclusions may be mistakenly thought to be invalid, and invalid arguments with plausible conclusions may be mistakenly thought to be valid. This is known as the belief bias effect in psychology (Evans, Barston, \& Pollard, 1983; Newstead, Pollard, Evans, \& Allen, 1992; Oakhill \& Johnson-Laird, 1985; Revlin, Leirer, Yop, \& Yop, 1980). On the other hand, to the extent that one's reasoning strategy favors logic, one should be willing or able to ignore the empirical content of the conclusion and simply evaluate the argument in terms of its logical structure. This study was a first investigation into possible cultural differences in this robust phenomenon. It was expected that Koreans would show a stronger belief bias than European Americans. Furthermore, this cultural difference in belief bias was expected to emerge holding logical reasoning ability constant, as measured by performance on the abstract arguments.

\section{Method}

\section{$\underline{\text { Participants }}$}

Eighty-seven European American students at the University of Michigan (35 men, 52 women, age $\underline{\mathrm{M}}=19$ ) and 99 Korean students at Yonsei University in Seoul, Korea (71 men, 28 women, age $\underline{M}=23$ ) participated in this study for partial course credit. In addition, a separate 
group of 30 European Americans (12 men, 18 women, age $\underline{\mathrm{M}}=19)$ and 30 Koreans (18 men, 12 women, age $\underline{\mathrm{M}}=22$ ) rated the believability of the conclusions as a manipulation check.

Materials

Argument validity was crossed with conclusion believability, generating 4 arguments for each of the 4 following argument types: valid/believable, valid/nonbelievable, invalid/believable, invalid/nonbelievable (see Table 3 for examples). After participants evaluated the 16 arguments, they were asked to evaluate additional 8 arguments having the same logical structure as the prior arguments except that they were presented in abstract form, and hence believability was irrelevant. These abstract arguments were instantiated by letters and foreign unfamiliar words. Thus, each participant evaluated a total of 24 arguments.

Each type of argument as described above in turn consisted of four kinds of valid logical forms and their invalid counterparts that varied in difficulty: Modus Ponens (All A are B. C are A. C are B.), Modus Tollens (All A are B. C are not B. C are not A.), Modus Tollens conditional arguments conforming to a permission schema (If A is B, it may get C. $\mathrm{X}$ is not permitted to get C. X is not B.), and an argument of the form: No A are B. Some C are B. Some C are not A. Procedure

The study was conducted in a classroom setting. Participants received a questionnaire packet containing the 24 syllogistic and conditional arguments. The concrete arguments consisting of the 4 different types were listed in a scrambled order, followed by the abstract arguments, also in scrambled order. For the Korean participants, all materials were translated into Korean using the back-translation method (Brislin, 1970). Participants were given as much time as they needed, with all participants completing the study within 20 minutes. 
Participants were instructed to evaluate whether or not the conclusion followed logically from the premises for each argument (they had to circle either yes or no). Unlike in Study 3 in which participants were not made aware that the task could be solved by applying logic, in this study, strong instructions were presented that explicitly encouraged logical reasoning:

In this study, you are going to see a series of problems. In each problem, you must decide whether the stated conclusion follows logically from the premises or not. You must suppose that the premises are true and limit yourself only to information contained in these premises. Do not be concerned if some of the terms in some of the problems seem unfamiliar to you...For each problem, decide if the given conclusion follows logically from the premises. Circle YES if, and only if, you judge that the conclusion can be derived from the given premises. Otherwise circle NO. You can take your time to answer each problem. However do not spend too much time on any single problem.

The dependent measure was each participant's percentage of "yes" responses for each argument type, indicating whether or not the participant thought the conclusion of the argument did follow logically from the premises.

A separate group of European American and Korean students rated the believability of the conclusions, as a manipulation check for believability, and in order to establish the functional equivalence of the believability information for both cultures. Participants rated the conclusions of the 16 concrete arguments, deciding their believability on a scale anchored between -3 (very nonbelievable) and +3 (very believable), with zero being neither believable nor nonbelievable. 


\section{Manipulation Check}

\section{$\underline{\text { Results }}$}

The believability manipulation was successful for both cultures: European Americans and Koreans agreed that believable conclusions were indeed believable and nonbelievable ones were indeed nonbelievable. Averaging across all 8 believable and 8 nonbelievable conclusions, European Americans rated believable conclusions, $\underline{\mathrm{M}}=2.12, \underline{\mathrm{SD}}=.58$, which is significantly different from zero, $\underline{\mathrm{t}}(29)=19.92, \underline{\mathrm{p}}<.001$, and they rated nonbelievable conclusions, $\underline{\mathrm{M}}=-2.12$, $\underline{\mathrm{SD}}=.43$, which is also different from zero, $\underline{\mathrm{t}}(29)=27.39, \underline{\mathrm{p}}<.001$. Similarly, Koreans' ratings of believable and nonbelievable arguments were different from zero, $\underline{\mathrm{M}}=1.29, \underline{\mathrm{SD}}=.79$, $\underline{\mathrm{t}}(29)=$ 8.93, $\underline{\mathrm{p}}<.001$, and $\underline{\mathrm{M}}=-2.20, \underline{\mathrm{SD}}=.47, \underline{\mathrm{t}}(29)=25.80, \underline{\mathrm{p}}<.001$, respectively.

Although the ratings of European Americans and Koreans did not differ for nonbelievable conclusions, $\underline{\mathrm{t}}<1$, Koreans found believable conclusions to be significantly less believable than did European Americans, $\mathfrak{t}(58)=4.63, \underline{p}<.001$. The weaker Korean commitment to the believable conclusions works against finding support for the hypothesis of this study (for invalid arguments), which predicts a stronger belief bias for Koreans. Results consistent with this prediction, therefore, could not be due to differential commitment to the believable conclusions.

\section{$\underline{\text { Abstract Arguments }}$}

Figure 7 presents the results for the abstract arguments. There was evidence for response bias, such that Koreans were overall less likely to respond "yes", $\underline{F}(1,184)=14.41, \underline{p}<.001$. To evaluate performance in discriminating valid from invalid arguments while controlling for response bias, a single measure of accuracy was computed: hits (percentage of "Yes" responses for valid arguments) minus false alarms (percentage of "Yes" responses for invalid arguments). There was no cultural difference in accuracy, $\underline{\mathrm{t}}<1, \underline{\mathrm{M}}=45.1 \%$ and $\underline{\mathrm{M}}=48.5 \%$ for European Americans and 
Koreans, respectively. Consistent with this pattern, there was no culture by argument validity interaction, $\underline{\mathrm{F}}<1$. Thus any cultural differences found in belief bias cannot be attributed to differences in logical reasoning ability.

As can be seen, participants' reasoning, regardless of culture, tracked the logical structure of the arguments. European Americans and Koreans rated valid arguments as valid at significantly above chance (i.e., $50 \%), \underline{\mathrm{t}}(86)=17.34, \underline{\mathrm{p}}<.001$, and $\mathrm{t}(98)=11.42, \underline{\mathrm{p}}<.001$, respectively.

Similarly, they rated invalid arguments as not valid at significantly above chance, $\underline{t}(86)=4.61, \underline{p}<$ .001 , and $\mathrm{t}(98)=9.75, \mathrm{p}<.001$, respectively.

\section{$\underline{\text { Concrete Arguments }}$}

We anticipated that Koreans, relative to European Americans, would be more likely to evaluate the arguments as valid when the conclusion is believable, and less likely to do so when the conclusion is nonbelievable. A culture by argument validity by conclusion believability ANOVA revealed a main effect of argument validity $\underline{\mathrm{F}}(1,184)=1161.73, \underline{\mathrm{p}}<.001$, indicating sensitivity to logical structure; conclusion believability, $\underline{\mathrm{F}}(1,184)=90.45, \mathrm{p}<.001$, indicating a belief bias; and culture, $\underline{\mathrm{F}}(1,184)=26.91, \mathrm{p}<.001$, indicating a response bias (discussed below). Critically, there was a 3-way interaction between culture, argument validity, and conclusion believability, $\underline{\mathrm{F}}(1,184)=4.73, \underline{\mathrm{p}}<.05$. This 3-way interaction reflects the fact that the belief bias was stronger for valid than invalid arguments ${ }^{2}$, and that it was larger for Koreans than for Americans, but only for valid arguments. Therefore, the belief bias effect was examined separately for valid and invalid arguments, with culture as a between-groups factor, and conclusion believability as a within-groups factor.

Valid Arguments. As may be seen in the top panel of Figure 8, the predicted interaction was obtained: Koreans showed a stronger belief bias effect than Americans, $\underline{F}(1,184)=4.31, \underline{p}$ 
$<.05$. Overall, there was a belief-bias effect across cultures, $\underline{\mathrm{F}}(1,184)=86.45, \underline{\mathrm{p}}<.001$. As in abstract arguments, there was a difference in response bias such that Koreans were overall less likely to respond "yes," $\underline{F}(1,184)=11.82, \underline{p}<.002$.

Invalid arguments. Contrary to predictions, no culture by conclusion believability interaction emerged for invalid arguments, $\underline{\mathrm{F}}<1$ (bottom panel of Figure 8). The belief bias effect was replicated for invalid arguments, $\underline{\mathrm{F}}(1,184)=25.27, \underline{\mathrm{p}}<.001$. The response bias across cultures emerged again, $\underline{\mathrm{F}}(1,184)=13.43, \mathrm{p}<.001^{3}$.

As was the case with abstract arguments, overall performance in the concrete arguments was well calibrated to the logical structure of the concrete arguments. All 8 comparisons of the means to the chance level were statistically significant at $\mathrm{p}<.001$ for both European Americans and Koreans, for both believable and nonbelievable arguments.

\section{$\underline{\text { Discussion }}$}

In sum, Koreans showed a stronger belief bias effect than European Americans, though only for valid arguments. Because European Americans and Koreans showed the same degree of logical reasoning ability for the abstract arguments, we can conclude that the stronger belief bias of Koreans was not due to differences in reasoning ability, but rather to differing tendencies to decontextualize the content of an argument from its logical structure.

Why did we obtain a cultural difference in belief bias for valid but not invalid arguments? Recall that in the manipulation check, separate groups of Koreans and Americans judged the plausibility of the believable and nonbelievable conclusions. Both cultural groups found the nonbelievable conclusions equally implausible. However Koreans found the believable conclusions on average less plausible than did the Americans. Thus the same believable conclusions--contained in the invalid arguments--were weaker for Koreans than for Americans. Since Koreans had a 
weaker belief only in the invalid arguments, they may have been less influenced by beliefs in the invalid arguments. As a result, belief bias for the invalid arguments was not stronger for Koreans.

\section{General Discussion}

The present research investigated cultural preferences for formal and intuitive reasoning. Formal reasoning is rule-based, emphasizes logical inference, represents concepts by necessary and sufficient features, and overlooks sense experience when it conflicts with rules or logic. Intuitive reasoning is experience-based, resists "decontextualizing" or separating form from content, relies on sense experience and concrete instances, and overlooks rules and logic when they are at odds with intuition.

We compared the reasoning of European American, Asian American, and East Asian university students, under conditions where a cognitive conflict was activated between formal and intuitive strategies of thinking. The central hypothesis was that European Americans would be more willing to set aside intuition and follow rules than East Asians.

Study 1 showed that when a complex rule was in conflict with exemplar memory, European Americans made fewer classification errors in category learning than East Asians. European Americans were also faster in rule application than East Asians. Learning based on exemplars, in contrast, was entirely similar for East Asians and European Americans. Study 2 examined classification in which a simple rule was pitted against family resemblance structure. When participants were asked to assign the object to a category, all participants, regardless of culture, made their assignments based on the rule, consistent with prior research (e.g., Medin et al., 1987). However, when instructions encouraged attention to all properties of the categories by judging the similarity of the object to the categories, East Asians' responses were most often based on family resemblance, whereas those of European Americans were still based on the rule. 
Studies 3 and 4 examined thought processes that were less driven by immediate perception, and relied on pre-existing concepts and beliefs. Study 3 pitted logic against exemplar typicality. Participants rated the convincingness of deductive categorical arguments with either typical or atypical conclusions. Koreans showed a strong typicality effect, whereas European Americans produced responses that were consistent with logic. Moreover, Koreans were equally affected by typicality whether or not typicality was made salient; European Americans were affected by typicality only when it was salient. Finally, Study 4 investigated deductive reasoning, pitting argument validity against conclusion believability. Whereas Americans and Koreans showed similar deductive reasoning for abstract arguments, Koreans showed a stronger belief bias effect (Revlin et al., 1980) for valid concrete arguments. (The same cultural difference did not occur, however, for invalid arguments.)

The cultural differences emerged only when formal and intuitive strategies were in conflict. There were no differences when participants were instructed simply to implement an intuitive strategy (exemplar-based categorization in Study 1), or a formal strategy (abstract deductive reasoning in Study 4). Although in each study we strived to rule out obvious methodological artifacts, it is possible that each individual finding could be subject to an alternative explanation. For this reason, we note that each specific cultural difference reported in this paper should be interpreted with caution. However, our strategy of seeking converging evidence from four rather different paradigms, using different sets of instructions, tasks, stimuli, and cultural samples suggests that the best explanation for the cognitive differences that emerged in these studies is that there are different cultural preferences for the use of cognitive strategies to solve the same problem. 
In all three studies in which they were included, Asian Americans were either similar to European Americans, or intermediate between European Americans and East Asians. This finding perhaps reflects a substantial though not complete socialization of Asian Americans into the Western mode of thought. Because Asian Americans are exposed to many of the same extrafamilial experiences as European Americans (in that they largely experience the same societal and educational environment), but are likely to differ in the kinds of intrafamilial and perhaps peer socialization they receive, this finding suggests that intellectual socialization is at least partly due to proximal influences ${ }^{4}$.

\section{Possible Social Origins of the Cultural Differences in Reasoning}

Why are Western and East Asian participants biased towards different modes of reasoning? The attempt to answer this important question must of course be speculative at this time because it involves complex sociological and historical issues beyond the scope of this paper, and we leave it as an open question for the future. Here we mention briefly three factors that have been identified by philosophers, ethnographers, and social historians (for a fuller discussion, see Nisbett, et al., 2001). First, the practice of adversarial debate prevalent in Western cultures, as opposed to the practice of consensus-based decision making prevalent in East Asia have been linked to analytic versus holistic cognitive orientations, respectively (e.g., Lloyd, 1990; Ohbuchi and Takahasi, 1994). Second, pedagogical practices emphasizing critical thinking in Western classrooms, as opposed to experience-based learning in Chinese classrooms appear to encourage differing modes of thinking in Western and East Asian societies (On, 1996; for a review, see Tweed \& Lehman, in press). Third, many historians of science and philosophy both Western and East Asian have reported important cultural differences in the dominant philosophical traditions that have influenced the intellectual practices in the West and East. Early Greek and Chinese 
philosophy, science, and mathematics were quite different in their strengths and weaknesses. Many Greek philosophers looked for universal rules to explain events and were concerned with categorizing objects with precision and with respect to their "essences." There was a marked distrust of intuition. Chinese philosophers, especially Taoists, were more pragmatic and intuitive, and were distrustful of formal logic and rational distinctions (Fung, 1952; Liu, 1974; Lloyd, 1990, 1996; Nakamura, 1960/1988; Needham, 1954; Russel, 1945; for a discussion of the evidence, see Norenzayan, 1999).

We found cognitive differences in reasoning that to some degree mirror differences in philosophical traditions. As provocative as this congruence may be, we cannot know at this time if these traditions are actually implicated in such reasoning processes. Whether or not there is psychological continuity between these philosophical traditions and reasoning processes remains an open question. The findings reported in this paper and elsewhere (see Nisbett, et al.) can serve as one starting point for such interdisciplinary investigation.

\section{Normative Considerations: Reasoning Strategies as Tools for Thought}

Although a full examination of normative issues is beyond the scope of this paper, it is nevertheless important to mention several normative considerations.

A general point about analytic and holistic thinking is that each mode of thought produces a mixed bag of normative and non-normative outcomes. For example, in deductive reasoning, analytic thinkers tend to respond more logically than holistic thinkers, but in causal attribution, the former more readily commit the fundamental attribution error than the latter. Conversely, holistic thinkers are more accurate in covariation detection (Ji, Peng, \& Nisbett, in press), yet they are more vulnerable to hindsight bias (Choi \& Nisbett, 2000). Thus, neither the analytic nor the holistic mode guarantees accuracy in reasoning. 
In two studies presented here, the East Asian reliance on intuitive processes led to less accurate responses than the American reliance on rule-based approaches. In Study 1, East Asians made more errors when asked to categorize the negative matches--for which a conflict existed between rule-based and exemplar-based judgments. We note, however, that under other task conditions intuitive responses could lead to more accurate responses than would formal processes. One circumstance that favors reliance on an intuitive strategy occurs when a rule is only partially predictive, like many rules in everyday life. For example, in the category learning procedure of Study 1, suppose the rule were made only partially predictive of the categories, such that it would accurately predict category membership for positive match animals, but would predict the wrong category for negative match animals. In such a situation, it has been shown that switching to an exemplar-based strategy leads to superior overall performance than continuing to use the rule (e.g., Allen \& Brooks, 1991, Experiment 4), and East Asians might perform more accurately than European Americans (a proposal that remains to be tested).

East Asians also made more errors in Study 4, in which they were explicitly asked to judge the logical validity of the concrete arguments, as opposed to their plausibility or convincingness. Though this is an error, and undoubtedly one that would have been acknowledged by the participants themselves given their performance with fully abstract materials, it is the result of a bias that does not always yield less reasonable conclusions than the rationalistic bias of Westerners. Indeed, many East Asian scholars have noted that in East Asian cultures logic does not enjoy the normative status that it does in the West. In Japanese culture, for example, "to argue with logical consistency is thus discouraged, and if one does so continuously one may not only be resented but also be regarded as immature (Nagashima, 1973, p. 96). Liu (1974) makes a similar point about China when he writes, “...it is precisely because the Chinese mind is so rational that it 
refuses to become rationalistic and ... refuses to separate form from content" (p. 325). Consistent with these assertions, recent evidence indicates that Koreans rank "being an intuitive person" to be important for work success more than Americans, whereas the opposite is true for "being a logical person” (Norenzayan \& Sanchez-Burks, 2002). Furthermore, in some of the research conducted by our colleagues, European Americans have been shown to make errors, in their efforts to be logically consistent, that actually result in judgments that are incoherent in the sense that one judgment actually follows from the opposite of the other (Peng \& Nisbett, 1999; Davis, Nisbett, \& Schwarz, 2000). These errors were avoided by East Asian participants, who, however, made logical errors of their own in their attempts to reconcile opposing views.

The philosopher Stephen Stich (1990) has said that "there are no intrinsic epistemic virtues...cognitive mechanisms or processes are to be viewed as tools or policies and evaluated in much the same way that we evaluate other tools or policies" (1990, p. 24). If there are culturally diverse ways to go about the business of cognition, and if there are culturally diverse systems of justification which serve the needs of various cognitive communities, then the reasonable philosophical position would be to evaluate thinking in terms of the local standards of justification, as well as specific task requirements (Stich, 1990; see also Resnick, 1994). This is not to say that it is unreasonable to criticize specific inferential practices, even by the standards of another culture, but just that criticism must be cognizant both of inferential goals and cultural context. 


\section{References}

Ahn, W., \& Medin, D. L. (1992). A two-stage model of category construction. $\underline{\text { Cognitive }}$ Science, 16, 81-121.

Allen, S. W., \& Brooks, L. R. (1991). Specializing in the operation of an explicit rule. Journal of Experimental Psychology: General, 120, 3-19.

Arnheim, R. (1969). Visual thinking. Berkeley, CA: University of California Press.

Atran, S. (1990). Cognitive foundations of natural history. New York: Cambridge University Press.

Brislin, R. W. (1970). Back-translation for cross-cultural research. Journal of CrossCultural Psychology, 1, 185-216.

Chaiken, S., \& Trope, Y. (Eds.). (1999) Dual-process theories in social psychology . New York: Guilford Press.

Choi, I., \& Nisbett, R. E. (2000). The cultural psychology of surprise: Holistic theories and recognition of contradiction. Journal of Personality and Social Psychology, 79 890-905.

Choi, I., Nisbett, R. E., \& Norenzayan, A. (1999). Causal attribution across cultures: Variation and universality. Psychological Bulletin, 125, 47-63.

Cole, M., Gay, J., Glick, J. A., \& Sharp, D. W. (1971). The cultural context of learning and thinking: An exploration in experimental anthropology. New York: Basic Books.

Davis, M., Nisbett, R. E., \& Schwarz, N. (2000). Responses to weak arguments by East Asians and Americans. Ann Arbor: University of Michigan.

Evans, J. B. T., Barston, J. L., \& Pollard, P. (1983). On the conflict between logic and belief in syllogistic reasoning. Memory and Cognition, 11, 295-306. 
Evans, J. B. T., \& Handley, S. J., Harper, C. N. J., \& Johnson-Laird, P. N. (1999).

Reasoning about necessity and possibility: A test of the mental model theory of deduction. Journal of Experimental Psychology:Learning, Memory, \& Cognition, 25, 1495-1513.

Evans, J. B. T., \& Over, D. E. (1996). Rationality and reasoning. Hove: Psychology Press.

Fischhoff, B. (1975). Hindsight's foresight: The effect of outcome knowledge on judgment under uncertainty. Journal of Experimental Psychology: Human Perception and Performance, 1, 288-299.

Fung, Y. L. (1952). A history of Chinese philosophy (D. Bodde, Trans.). (Vol. I). Princeton, NJ: Princeton University Press.

Gentner, D., \& Medina, J. (1998). Similarity and the development of rules. Cognition, 65, 263-297.

Gilbert, D.T. (1991). How mental systems believe. American Psychologist, 46, 107-119.

James, W. (1890). The principles of psychology. New York: Dover Publications.

Ji, L., Peng, K., \& Nisbett, R. E. (in press). Culture, control, and perception of relationships in the environment. Journal of Personality and Social Psychology.

Kashima, Y., Yamaguchi, S., Kim, U., Choi, S., Gelfand, M. J., \& Yuki, M. (1995). Culture, gender, and self: A perspective from individualism-collectivism research. Journal of Personality and Social Psychology, 69, 925-937.

Keil, F. (1994). The birth and nurturance of concepts by domains: The origins of concepts of living things. In L. Hirschfeld \& S. Gelman (Eds.), Mapping the mind: Domain specificity in cognition and culture. New York: Cambridge University Press.

Kemler-Nelson, D. G. (1984). The effect of intention on what concepts are acquired. Journal of Verbal Learning and Verbal Behavior, 23, 734-759. 
Liu, S. H. (1974). The use of analogy and symbolism in traditional Chinese philosophy. Journal of Chinese Philosophy, 1, 313-338.

Lloyd, G. E. R. (1990). Demystifying mentalities. New York: Cambridge University Press. Lloyd, G. E. R. (1996). Science in antiquity: the Greek and Chinese cases and their relevance to the problems of culture and cognition. In D. R. Olson \& N. Torrance (Eds.), Modes of thought: Explorations in culture and cognition (pp. 15-33). Cambridge: Cambridge University Press.

Luria, A. (1971). Towards the problem of the historical nature of psychological processes. International Journal of Psychology, 6, 259-272.

Medin, D. L., Wattenmaker, W. D., \& Hampson, S. E. (1987). Family resemblance, conceptual cohesiveness, and category construction. Cognitive Psychology, 19, 242-279.

Nagashima, N. (1973). A reversed world: Or is it? In R. Horton, \& R. Finnegan (Eds.), Modes of thought: Essays on thinking in Western and non-Western societies (pp. 92-111). London: Faber and Faber.

Nakamura, H. (1960/1988). The ways of thinking of eastern peoples. New York: Greenwood Press.

Needham, J. (1954). Science and civilization in China (Vol 1). Cambridge, England: Cambridge University Press.

Neisser, U. (1963). The multiplicity of thought. British Journal of Psychology, 54, 1-14.

Newstead, S. E., Pollard, P., Evans, J. B. T., \& Allen, J. L. (1992). The source of the

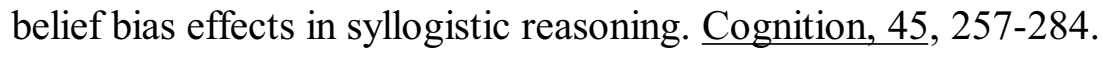


Nisbett, R.E., \& Norenzayan, A. (in press). Culture and cognition. In D. L. Medin (Ed.), Stevens' Handbook of Experimental Psychology: Cognition (3d Ed). New York: John Wiley \& Sons.

Nisbett, R. E., Peng, K., Choi, I., \& Norenzayan, A. (2001). Culture and systems of thought: Holistic vs. analytic cognition. Psychological Review, 108, 291-310.

Norenzayan, A. (1999). Rule-based and experience-based thinking: The cognitive consequences of intellectual traditions. Unpublished dissertation, University of Michigan, Ann Arbor.

Norenzayan, A., \& Nisbett, R. E. (2000). Culture and causal cognition. Current Directions in Psychological Science, 9, 132-135.

Norenzayan, A., \& Sanchez-Burks, J. (2002). A time for logic and a time for intuition: Normative evaluations of reasoning styles across cultures and contexts. Unpublished manuscript, University of Illinois, Urbana-Champaign.

Oakhill, J. V., \& Johnson-Laird, P. N. (1985). The effects of belief on the production of syllogistic conclusions. Quarterly Journal of Experimental Psychology, 37A, 553-569.

Ohbuchi, K. I., \& Takahashi, Y. (1994). Cultural styles of conflict management in Japanese and Americans: Passivity, covertness, and effectiveness of strategies. Journal of Applied Psychology, 24, 1345-1366.

On, L. W. (1996). The cultural context of Chinese learners: Conception of learning in the Confucian tradition. In D. A. Watkins \& J. B. Biggs (Eds.), The Chinese learner: Cultural, psychological, and contextual influences (pp. 25-42). Hong Kong: Comparative Education Research Centre. 
Peng, K., \& Nisbett, R. E. (1999). Culture, dialectics, and reasoning about contradiction. American Psychologist, 54, 741-754.

Regehr, G., \& Brooks, L. R. (1993). Perceptual manifestations of an analytic structure: The priority of holistic individuation. Journal of Experimental Psychology: General, 122, 92-114.

Resnick, L. B. (1994). Situated rationalism: Biological and social preparation for learning. In L. A. Hirschfeld \& S. A. Gelman (Eds.), Mapping the mind: Domain specificity in cognition and culture. Cambridge: Cambridge University Press.

Revlin, R., Leirer, V., Yop, H., \& Yop, R. (1980). The belief-bias effect in formal reasoning: The influence of knowledge on logic. Memory and Cognition, 8 , 584-592.

Rips, L. J. (1989). Similarity, typicality, and categorization. In S. Vosniadou \& A. Ortony (Eds.), Similarity and analogical reasoning. Cambridge: Cambridge University Press.

Rosch, E. (1978). Principles of categorization. In E. Rosch \& B. B. Lloyd (Eds.), $\underline{\text { Cognition and categorization. Hillsdale, NJ: Erlbaum. }}$

Rosch, E., \& Mervis, C. B. (1975). Family resemblances: Studies in the internal structure of categories. Cognitive Psychology, 7, 573-605.

Ross, L. (1977). The intuitive psychologist and his shortcomings. In L. Berkowitz (Ed.), Advances in experimental social psychology (Vol. 10, ). New York: Academic Press.

Russell, B. (1945). A history of Western philosophy. New York: Simon \& Schuster. Scribner, S. (1977). Modes of thinking and ways of speaking: Culture and logic reconsidered. In P. Johnson-Laird \& P. Wason (Eds.), Thinking: Readings in cognitive science (pp. 483-500). New York:: Cambridge University Press.

Sloman, S. (1993). Feature-based induction. Cognitive Psychology, 25, 231-280. 
Sloman, S. (1996). The empirical case for two systems of reasoning. Psychological Bulletin-, 119, 30-22.

Smith, E. E., Langston, C., \& Nisbett, R. E. (1992). The case for rules in reasoning. Cognitive Science, 16, 1-40.

Smith, E. E., \& Medin, D. L. (1981). Categories and Concepts. Cambridge, MA: Harvard University Press.

Smith, E. E., Patalano, A. L., \& Jonides, J. (1998). Alternative strategies of categorization. Cognition, 65, 167-196.

Smith, J. D., \& Kemler, D. G. (1984). Overall similarity in adults' classification: The child in all of us. Journal of Experimental Psychology: General, 113, 137-159.

Stanovich, K. E. (1999). Who is rational? Studies of individual differences in reasoning. Mahwah, NJ: Lawrence Erlbaum Associates.

Stich, S. P. (1990). Fragmentation of reason. Cambridge, MA: MIT Press.

Tversky, A., \& Kahneman, D. (1983). Extensional versus intuitive reasoning: The conjunction fallacy in probability judgment. Psychological Review, 90, 293-315.

Tweed, R. G., \& Lehman, D. R. (in press). Learning considered within a cultural context: Confucian and Socratic approaches. American Psychologist.

Witkin, H. A., \& Berry, J. W. (1975). Psychological differentiation in cross-cultural perspective. Journal of Cross Cultural Psychology, 6, 4-87.

Wittgenstein, L. (1953). Philosophical investigations. New York: Macmillan. 


\section{Author Note}

This research is based on a doctoral dissertation submitted by Ara Norenzayan under the guidance of Richard Nisbett to the University of Michigan. Scott Atran, Jonathan Evans, Eric Lormand, Norbert Schwarz, and Yingrui Yang provided valuable comments on an earlier draft. We thank Viola Chen, Jason Drwal, Sally Harrison, Young Ho Hong, Jong Dae Kim, Yookyong Lee, Mehyun Song, and Miguel Wong for their assistance in conducting this research, and Andrea Patalano for providing the stimuli for Study 1.

This research was supported by a Rackham Predoctoral Fellowship of the University of Michigan and an NSF Graduate Research Fellowship to Ara Norenzayan, and by an NSF grant (SBR 9729103) to Richard Nisbett.

Address correspondence to Ara Norenzayan, Department of Psychology, University of Illinois at Urbana-Champaign, 603 East Daniel Street, Champaign, IL 61820. 


\section{Footnotes}

${ }^{1}$ In this study, typicality describes a kind of intuitive reasoning distinct from family resemblance. Typicality is used to refer to peoples' intuitions about the "goodness" of naturally occurring exemplars, whereas family resemblance is objectively determined as the proportion of features shared among category members, as in Study 2.

${ }^{2}$ That the belief bias was larger for valid than invalid arguments is surprising. Typically, the belief bias tends to be larger for invalid than valid arguments (Evans \& Over, 1996; Newstead et al., 1992). However, this interaction can be reliably eliminated, and even non-significantly reversed, when (1) strong instructions are used that emphasize logical reasoning and logical necessity, or (2) when the invalid arguments are determinately invalid, that is, invalid because the conclusion definitely fails to follow from the premises. In contrast, indeterminately invalid arguments are invalid because the premises give insufficient information to judge whether the conclusion holds or not (Evans, Handley, Harper, \& Johnson-Laird, 1999; for a discussion, see Newstead et al., 1992). Both of these factors were simultaneously present in this study. That is, very strong logical instructions were used, and 3 of the 4 invalid argument forms were determinately invalid. This could explain why the impact of belief was reversed, i.e., a stronger belief bias effect was found for valid arguments.

${ }^{3}$ Two of the four argument forms used in this study did not have "ALL" quantifiers for the second premise and the conclusion (second and third argument forms in Table 3). It is possible that this could have created quantifier ambiguity, i.e., interpreting them in terms of existential (SOME) instead of universal (ALL) quantifiers. Note, however, that there was no evidence in the solution rates to these particular arguments that this was the case. Nor is there any reason to believe that this potential ambiguity was any different for the two cultures. Nevertheless, we reanalyzed the 
data by considering only the argument forms that explicitly stated all the quantifiers (First and fourth argument forms in Table 3, for a total of 8 arguments). Almost identical results were obtained: there was a main effect of logic, $\underline{F}(1,184)=696.50, \underline{p}<.001$; a main effect of belief, $\underline{F}(1,184)=85.35, \underline{p}<.001 ;$ a main effect of culture, $\underline{F}(1,184)=26.72, \underline{p}<.001$ (reflecting the difference in response bias), and a 3 -way logic by belief by culture interaction, $\underline{F}(1,184)=5.17$, $\underline{p}$ $<.03$, indicating a stronger belief bias for Koreans, but only for valid arguments. Culture by belief interaction for valid arguments, $\underline{F}(1,184)=3.07, \underline{p}=.08$, and for invalid arguments, $\underline{F}(1,184)=$ $1.95, \mathrm{p}=\mathrm{ns}$.

${ }^{4}$ Although this research was not concerned with gender, post-hoc analyses were conducted to examine gender differences in thinking except for Study 1 (where there was an inadequate sample size). Overall, women were somewhat more likely to rely on intuitive strategies, whereas men were more likely to rely on formal rules. Importantly, the gender differences were orthogonal to the cultural differences. Also, the gender differences tended to be less reliable and smaller in size than the cultural differences. This pattern of cultural and gender differences reinforces prior research indicating that that there is greater psychological similarity among men and women of the same culture than people of different cultures who share the same gender (Kashima et al., 1995). The analyses for gender differences are available from the first author. 
Table 1. Category Structures in Study 2.

\begin{tabular}{ll}
\hline Group 1 & Group 2 \\
\hline 0000 & 1111 \\
0100 & 1011 \\
0010 & 1101 \\
0001 & 1110 \\
& \\
Target Objects & \\
0111 & \\
1000 & \\
\hline
\end{tabular}

Note. The two categories (groups) in each stimulus set were instantiated in four exemplars per group, varying on four binary features. The value of each binary feature is represented as 0 or 1 . Each row represents one exemplar, and each column represents the distribution of values for one feature. The first column corresponds to the defining rule. 
Table 2. Selected Arguments in Study 3.

Arguments with Typical Conclusions

1. All birds have an ulnar artery

Therefore, all eagles have an ulnar artery

2. All professionals do community service in Tahiti

Therefore, all doctors do community service in Tahiti

3. All clothing is made of Supsa leaves in the Island of Pago-Pago

Therefore, all shirts are made of Supsa leaves in the Island of Pago-Pago

4. All diseases can be understood in terms of the Shawinigan principle

Therefore all cancer can be understood in terms of the Shawinigan principle

\section{Arguments with Atypical Conclusions}

1. All birds have an ulnar artery

Therefore, all penguins have an ulnar artery

2. All professionals do community service in Tahiti

Therefore, all bankers do community service in Tahiti

3. All clothing is made of Supsa leaves in the Island of Pago-Pago

Therefore, all raincoats are made of Supsa leaves in the Island of Pago-Pago

4. All diseases can be understood in terms of the Shawinigan principle

Therefore all cholera can be understood in terms of the Shawinigan principle 
Table 3. Selected Arguments in Study 4.

$\underline{\text { Valid/Believable }}$

Premise 1: No police dogs are old

Premise 2: Some highly trained dogs are old

Conclusion: Some highly trained dogs are not police dogs

Valid/Nonbelievable

Premise 1: All things that are made of plants are good for the health

Premise 2: Cigarettes are things that are made of plants

Conclusion: Cigarettes are good for the health

Invalid/Believable

Premise 1: All tall athletes have large foot size

Premise 2: Famous basketball players have large foot size

Conclusion: Famous basketball players are tall athletes

Invalid/Nonbelievable

Premise 1: If a country is a member of the European Community, it is permitted to apply for loans from the European Bank

Premise 2: India is not permitted to apply for loans from the European Bank

Conclusion: India is a member of the European Community 


\section{Figure Captions}

Figure 1. Examples of stimuli (Study 1). Rule: Animal lives on VENUS if it has at least 3 of the following 5 features: curly tail, hooves, antennae ears, mouth, long neck. Otherwise it lives on SATURN.

Figure 2. Category learning: Mean classification error rates and reaction times for the exemplar memory group.

Figure 3. Category learning: Mean classification error rates and reaction times for the rule group. Figure 4. Examples of stimuli (Study 2). Each of the two target objects was presented separately with the two groups to achieve a counterbalanced design. For the flowers, the defining feature is the stem length; for the geometric figures, it is the top-most string.

Figure 5. Classification and similarity judgments by rule vs. family resemblance.

Figure 6. Convincingness of deductive arguments with typical vs. atypical conclusions, as a function of typicality salience.

Figure 7. Deductive reasoning with abstract arguments: Mean percent "valid" responses.

Figure 8. Deductive reasoning with concrete arguments having valid or invalid logical structure: Mean percent "valid" responses. 